\title{
THE CLOVER LEAF QUASAR H1413+117: NEW PHOTOMETRIC LIGHT CURVES
}

\author{
M. REMY ${ }^{1}$, E. GOSSET ${ }^{1,2}$ AND D. HUTSEMÉKERS ${ }^{1,2}$ \\ ${ }^{1}$ Institut d'Astrophysique, Liège, Belgium \\ ${ }^{2}$ Chercheur Qualifié au FNRS, Belgium \\ AND \\ B. REVENAZ ${ }^{3}$ AND J. SURDEJ ${ }^{3,4}$ \\ ${ }^{3}$ Space Telescope Science Institute, Baltimore, MD, USA \\ ${ }^{4}$ Directeur de Recherches au FNRS, Belgium
}

\begin{abstract}
Direct CCD images of the gravitationally lensed BAL quasar H1413+117 (Bessel B, V, R, I; Gunn r and i), obtained during 1989-1994 in the framework of photometric monitorings at ESO and with the NOT, have been carefully (re-)analyzed. Simultaneous fitting of 4 PSFs, fixing the relative positions of components $\mathrm{B}, \mathrm{C}$ and $\mathrm{D}$ with respect to $\mathrm{A}$, has been achieved successfully. The relative light curves of the 4 lensed QSO images, with respect to a stable nearby comparison star, confirm the general trend already presented by Arnould et al. (1993). The amplitude of the light curves is found to be about 0.5 magnitude, peak-to-peak in the $\mathrm{V}$ filter. The four lensed components display brightness variations quasi-simultaneously and in parallel. In addition to these intrinsic light variations due to the source, we find that component D shows extra light variations with respect to the other components. These are very likely caused by micro-lensing.
\end{abstract}

\section{Data, Photometry \& Discussion}

The optical photometry of the Clover Leaf BAL QSO, already presented in Arnould et al. (1993), has been carefully re-analyzed using multiple PSF fitting of the four lensed components. The relative magnitudes of the field stars with respect to the comparison star already used by Arnould et al. (1993) were checked. The scatter is of the order of 0.03 magnitudes $(3 \sigma)$. 


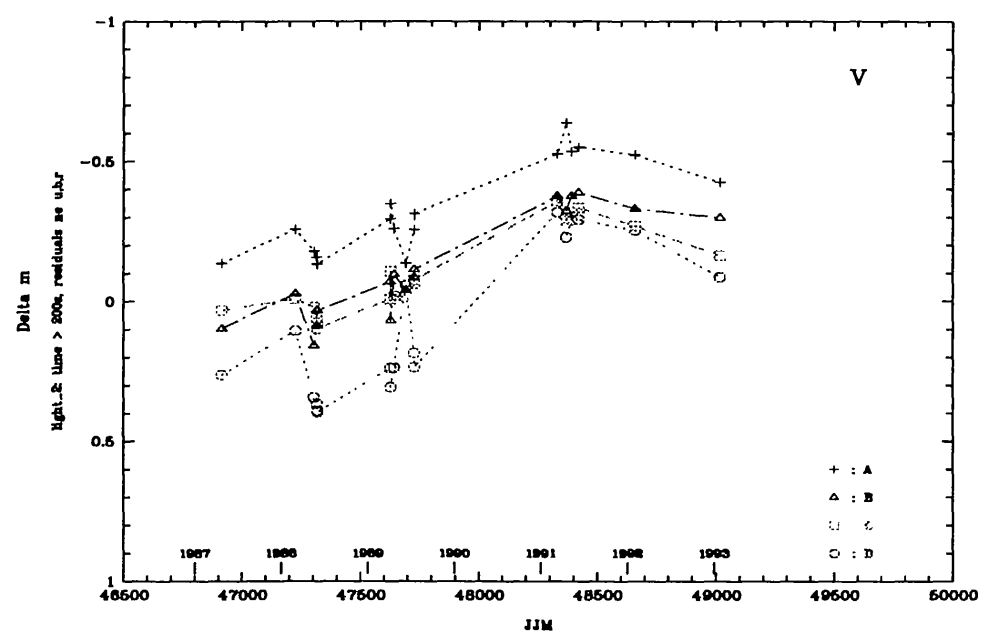

Figure 1. Photometry of $\mathrm{H} 1413+117$

The relative magnitudes in the $\mathrm{V}$ band of the four components of the Clover Leaf are plotted as a function of JD - 2400000.5 in Figure 1.

The qualitative trends already described by Arnould et al. (1993) appear more clearly in these plots, but some differences exist. The fluxes of the $\mathrm{A}, \mathrm{B}$ and $\mathrm{C}$ components vary in parallel and quasi-simultaneously with a peak in luminosity near 1991-93. The expected time delays between the four lensed components of this quasar are typically shorter than 1 month; intrinsic variations of the source would therefore show up quasi-simultaneously in Figure 1. The D component exhibits the same global trend but with additional light variations. Spectroscopic observations of the $\mathrm{D}$ component in March 1989 (Angonin et al. 1990) and June 1993 (with HST + FOS) show that while the continuum is clearly varying in agreement with the photometry, the superimposed emission lines are almost unchanged, providing further good evidence for a microlensing origin to the observed residual variations.

Acknowledgements: We would like to thank the numerous observers at ESO and the NOT. This research was partly supported by ESA under contract PRODEX.

\section{References}

Angonin, M.-C., et al., 1990, A\&A, 223, L5

Arnould, P., et al., 1993, in Gravitational Lenses in the Universe, eds. J. Surdej et al., (Liège: Université de Liège) 169 